\title{
Building defects and methodology of partial risk assessment as a step towards improving quality
}

\author{
Jaroslav Synek ${ }^{1}$, and Miloslava Popenková ${ }^{*}$ \\ ${ }^{1}$ Faculty of Civil Engineering, Department of Construction Technology, Czech Technical University \\ Prague, Thákurova 7, Prague and Metrostav, a. s., Koželužská 245, Prague 8, Czechia \\ ${ }^{2}$ Faculty of Civil Engineering, Department of Construction Technology, Czech Technical University \\ Prague, Thákurova 7, Prague, Czechia
}

\begin{abstract}
This article deals with the assessment of partial risks in construction projects. Partial risks compromise the quality of the construction during preparation, implementation and use. They are the cause of defects associated with individual parts of construction and supply. These risks are also a source of increased repair costs. We need to identify partial risks and suppress them at all stages of the construction project to improve quality and reduce construction costs. The article defines the sub-risk and its impact on the complexity of buildings. It deals with the methodology of evaluation and use of analytical results for the prevention of building defects. The article also presents the benefits of applying the sub-risk assessment and the resulting preventive measures.
\end{abstract}

\section{Introduction}

According to the available data, building projects are very often burdened by a bad reputation resulting from exceeding the implementation time and rising costs of projects that produce poor economic results. These are not just large projects, but also medium and small projects in all types of economies. The risks associated with these projects are the cause of poor results. Risk realization leads to building defects during preparation, implementation and use. Participants in building projects (investors, banks, designers, implementation firms, operators and users) should therefore be interested in improving the project's results and should therefore try to limit the realization of risks, the causes of the project's failure. In the long term, prevention is the most effective risk mitigation, ie procedures that prevent its implementation. However, routine risk management for the global risks of the whole project is not effective enough to ensure the quality of the individual parts of the project where individual risks to the quality of the buildings are realized. The work focuses on the limitation of operational risks, namely technical, technological risks associated with the development and use of technologies and manufacturing risks associated with production processes, not with other risks (contractual, financial, social, local, etc.). Therefore, in order to determine effective preventive

\footnotetext{
* Corresponding author: miloslava.popenkova@,fsv.cvut.cz
} 
procedures for individual parts of a building project, the risks and circumstances of their implementation must be analysed on the basis of defects analysis of parts of the construction (individual types of constructions, crafts and embedded technologies). The results of analyses use taxonomy, a list of defects, determining causes, as well as determining the magnitude of the consequences of a defect. Depending on the frequency and cost of defects in the evaluated production system, it is necessary to determine the risk values and subsequently determine the severity and extent of risk prevention. This is an effective process leading to the necessary reduction of potential losses and damage to construction projects.

\section{Targets}

The aim of the paper is to describe the already used methodology for identifying and evaluating the partial operational risks associated with the construction project and to clarify the procedures that can be used for effective prevention from the point of view of the contractor of the construction project. To prevent partial risks, it is necessary to focus on their identification and analysis, the procedures for their evaluation and the identification of the most serious of them.

\section{Definitions and characteristics of risk}

The general definition of risk is considered according to [1]: "The possibility that something unfavourable will happen in the future", also "Failure, Threat, Damage or Loss".

The risk for production processes can be understood as a threat, danger and uncertainty of the result. In the case of operational risks of construction projects, we consider the socalled technical risks as defined [2]: "The probable value of the loss incurred by the wearer, Risk recipients by realizing the hazard scenario, expressed in cash or other units."

This definition allows for an analytical, mathematical formulation describing the possible consequences for the implementation of the risks for the project. Risks can be defined as the probability of a threat or other negative event associated with a project endangering the achievement of its goals. If the overall objective of the project is the economic success (project profit), the completion of the required construction period and the proper functioning of the project, i.e. the quality (safety), the implementation of the risks threatens to achieve them. The risk is generally characterized by the probability of occurrence of a negative event (defect), the level of risk (high, medium, small), impacts (consequences), predictability, degree of responsiveness, relation to subject, rankings (primary, secondary, residual), size, acceptability, etc. (see taxonomy of risk [5]) and tables, as originals of good quality and well contrasted, are to be in their final form, ready for reproduction, pasted in the appropriate place in the text.

\section{Risk management, identification and classification and mathematics}

In order to achieve the objectives of the project (profit), it is necessary to actively control and control the risks based on their knowledge. This assumes the ability to evaluate them in relation to the goals and implementation practices used in the project. Own risk management includes their identification and classification (assessment), management plan, monitoring, risk response and risk communication. 


\subsection{Overall global risks}

Normally, the project's risks are generally assessed in relation to the project, site and conditions of implementation. These stem from an open environment of construction projects that require uninterrupted responses to the changing situation.

\subsection{Partial risks}

If we examine in detail the procedures needed to meet the project objectives, focusing on global general risks is not sufficient. For direct risk and quality management, it is necessary to focus on the quality / risks contained in the individual parts of the project, the components, and the technologies whose systemic partial quality / risks create or threaten the resulting quality of the project system. The project is to be understood as a system in accordance with the general theory of systems, i.e. as a system of elements and their interrelations, relations (see e.g. [3]).

Therefore, the basic task in risk management and ensuring the required quality of buildings is knowledge and way of eliminating partial quality / risks associated with the individual components (components) of buildings within the building - system. Construction is always a set, system of individual components and their elements. Only as a complex system they create the construction and its quality, respectively the quality of its use. For each partial part of the complex system - buildings are laid specific requirements, each part has specific features, requires specific requirements and conditions for assembly and processing, has specific conditions of use. The specific (partial) properties of the component are a condition for the proper functioning of the global building system as it is an indispensable part of the building system. The required functions of the system (the construction project) can be filled only if the complex system is working on the principle of conjunction, all required inputs must be filled in to meet the output requirements of the system, (quality) function of the building (e.g. maintaining the internal environment for the function) ) At the same time. If they are not filled, the response of the global system (output) does not meet the specified quality requirements, the system function is defective.

In order to achieve the specified quality, it is therefore necessary to focus on fulfilling the partial requirements for the quality of individual components, parts of the construction. This quality is necessary to require at all stages of the construction, from the formulation of the project, through the design, the realization to the construction. If this requirement is not met, the quality of the building as a system is jeopardized. Therefore, it is necessary to continuously check:

- Determination of partial component quality requirements from design to implementation

- Check for compliance with partial component quality requirements

- Running the building as a system in accordance with partial component quality requirements

The quality of the use of the building is a decisive criterion for quality assessment because the highest costs during the building life cycle are spent on building use, user maintenance and repairs. In order to achieve the necessary quality of use, it is therefore necessary for all members of the project team (investor, designer and contractor) to know and be able to work with the partial risks associated with the individual parts of the construction and their elements both in design and implementation. Risks need to be identified based on the knowledge of the results of the quality evaluation of previous projects. For identified defects, it is necessary to investigate and determine their causes (e.g. by category - ground, engineering, transport, etc.), to determine defective elements of the construction and their causes, the cost of their removal. 
The methodology should be based on current practices used to identify and assess risks [1] and use the specialized knowledge and information available to investors and building operators, designers and developers. This information should be shared by all participants in the construction project. Information on defects and disturbances is collected by participants in construction projects but rarely can be processed, archived and, most of all, actively used in the following projects. In some cases, such information is used systematically to improve new designs and practices. The aim of this methodology is to use the feedback possibilities to reduce design and implementation risks.

\section{Current status}

Risk analyses are currently used to assess the global risks of the entire construction project. Therefore, the risk is perceived as a risk of aggregation and such risk assessment does not provide sufficient information for the preventive measures of the implementing company related to the individual parts of the construction and the implementation processes. Therefore, there is no basis on how to prevent the realization of partial risks associated with individual parts of buildings and implementation processes. Methods of identification (risk assessment) of individual parts of buildings and processes (partial risks) are not well known and used.

The proposed procedures are aimed at suppressing and eliminating the individual risks directly related to parts of the construction and processes. These risks describe the differences in the threat to a certain part of the structure or process that may cause serious failures leading to permanent damage to structures and may require a reduction in load (structural requirements) or a change in design use as a result of reduced requirements. Permanent damage to the structure may result in additional reinforcement or rebuilding of the structure. Targeted prevention focused on the risk parts (processes) in preparation and implementation suppresses additional changes and repairs that are usually significant and costly. Therefore, it is necessary to analyse in detail the risks associated with individual parts and to implement these practices in the implementation practice. Ignoring this option brings companies involved in the endangered project a loss of reputation and loss of position on a highly competitive construction market.

\section{Methods}

The basis of the method of partial risk assessment is the use of feedback from the preparation of the construction and its implementation. The default information is the number of defects already encountered in the production system. The occurrence, frequency of failures is monitored for individual types of buildings (residential, administrative, production, transport, etc.) and their parts (supporting structure of the lower and upper structure, protection of the building against water, internal non-bearing structures, individual technological parts etc.) category. It is also necessary to identify the costs necessary to repair the defect (damaged structure or part of the building) and assign it to the individual categories (components). These costs are determined as an average at specified time or other intervals (e.g. calendar year, project), and in time series. The risk is then determined as the result of the observed frequency of occurrence and the long-term costs of removal of the defect.

Part of this method is to determine the causes of defects that use the information found to correct the defect. The causes are investigated at all stages in the design, implementation, even during the use of the building. Using partial risk assessments effectively reduces the high risks associated with design and implementation (e.g. large underground 
constructions). The method uses the information the contractor gains in evaluating the quality of the design of the building, the implementation processes and the information obtained during the use of the construction.

\section{Results}

The results of the partial risk assessment, in the context of a particular construction system of the building company (used construction techniques and technologies), are able to look for effective ways of limiting the risks associated with certain types, parts of buildings and building technologies used. Finding the critical parts of the building (construction) associated with critical risks, where it is necessary to focus on the risk reduction, increasing the resistance to defects allows the application of Pareto rule (80/20) [4]. Its application identifies those parts whose improvement will bring significant overall risk reduction and quality improvement. Preventive practices focus on individual components of the solution, identified parts, their formulation and parameters, technical solutions (design), manufacturing process techniques. Also included are the procedures used during the interoperational and final inspection of the construction, the use of the construction, its maintenance and repairs.

Detailed analysis of the parts and their relation to the complex function of the construction system is usually associated with feedback, sufficient information from the implementation and operation needed to find the method necessary to reduce the risk of defect to an acceptable boundary. Using Pareto rule on the most endangered parts of buildings will usually significantly reduce defects in the short term.

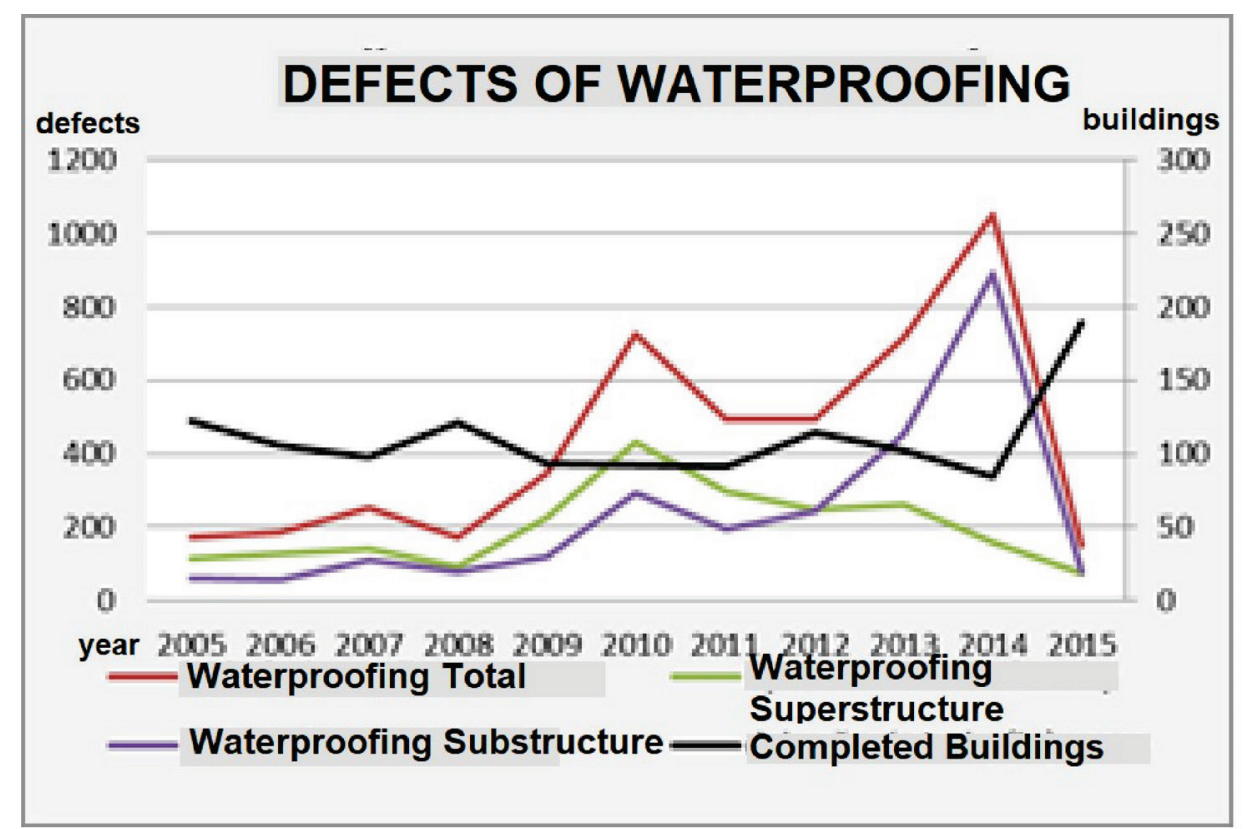

Fig. 1. Disturbance of Waterproofing of the Substructure - (number on right) red global waterproofing, green waterproofing of top structure, blue waterproofing of substructure, black number of completed building (on left).

An example is the application of this procedure to improve the quality of the substructure, water tightness. Insufficient water tightness of the substructure to groundwater 
impacts the construction mainly due to defects in the internal environment (leaks reduce the quality of the environment) and the formation of corrosion. The development of corrosion can mean, in the long term, a reduction in the bearing capacity and stability of the structure and thus its usefulness. It is therefore essential that the underground parts of the buildings are adequately protected from the negative effects of groundwater. Coating waterproofing usually applied to the outer surface of the structure is very sensitive to the correct design (overall water tightness), including penetrations (piping, etc.) and expansion of the structure. Coatings are susceptible to imperfect design and mechanical damage by other building activities (sharp fittings are installed just after a thin waterproofing from asphalt or plastic foils and can easily break through waterproofing). Water leaks often cause extensive defects in the indoor environment. These defects are difficult to locate and, above all, difficult to repair. Even after costly and extensive repairs to the substructure, they often carry permanent traces of damage, water leaks (even if they are limited) and, above all, the corrosion of the reinforcement.

For these reasons, procedures have been sought to significantly reduce these defect risks. All components of the solution have been examined and tested in relation to the manufacturing system. New specifications and requirements for substructure (parameters, proposed technical solutions, materials and products), technological procedures and control procedures for continuous and final quality control were formulated. As a result of the changes made to design and implementation, significant reductions in construction deficiencies, reduction of risks associated with permanent damage to the structure of corrosion and reduction of repairs were made.

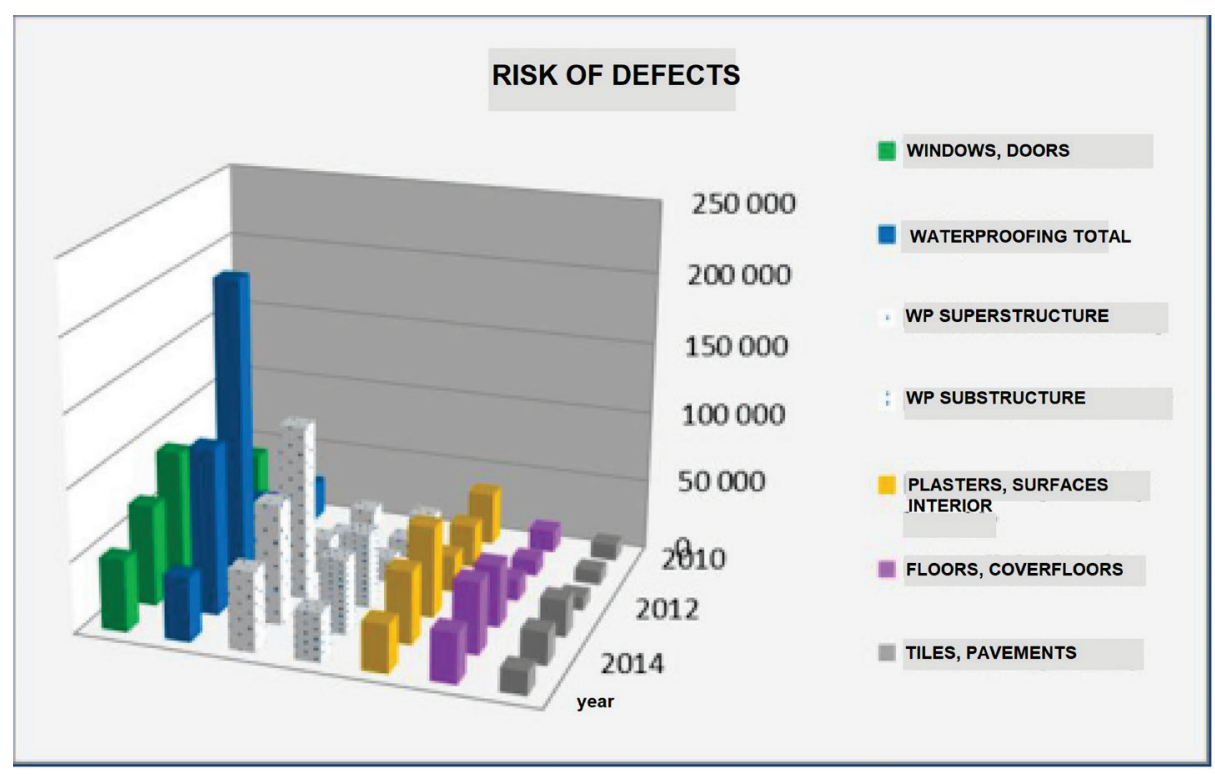

Fig. 2. Partial risks of structural defects y. 2010 - 14 (green windows and doors, blue waterproofing global, white and one blue point waterproofing of top structure, white and two blue point waterproofing of substructure, yellow surfaces, interior plaster, purple floors and floor coverings, grey tiling and paving). Volume of risks on right. 


\section{Conclusion}

Applying the described method of selecting parts of buildings with a high risk of damage allowed the focus on detailed analysis of defects of the part of the construction in all the components of the design, preparation and realization stages. The results of analyses identified the partial risks associated with the concept of the problem and the finding of effective preventive procedures to eliminate defects, i.e. to increase the resistance of the structure before the risks are realized. The application of the methodology brought a reassessment of the approach to evaluation of project proposals and methods of implementation and reduction of the number and extent of defects.

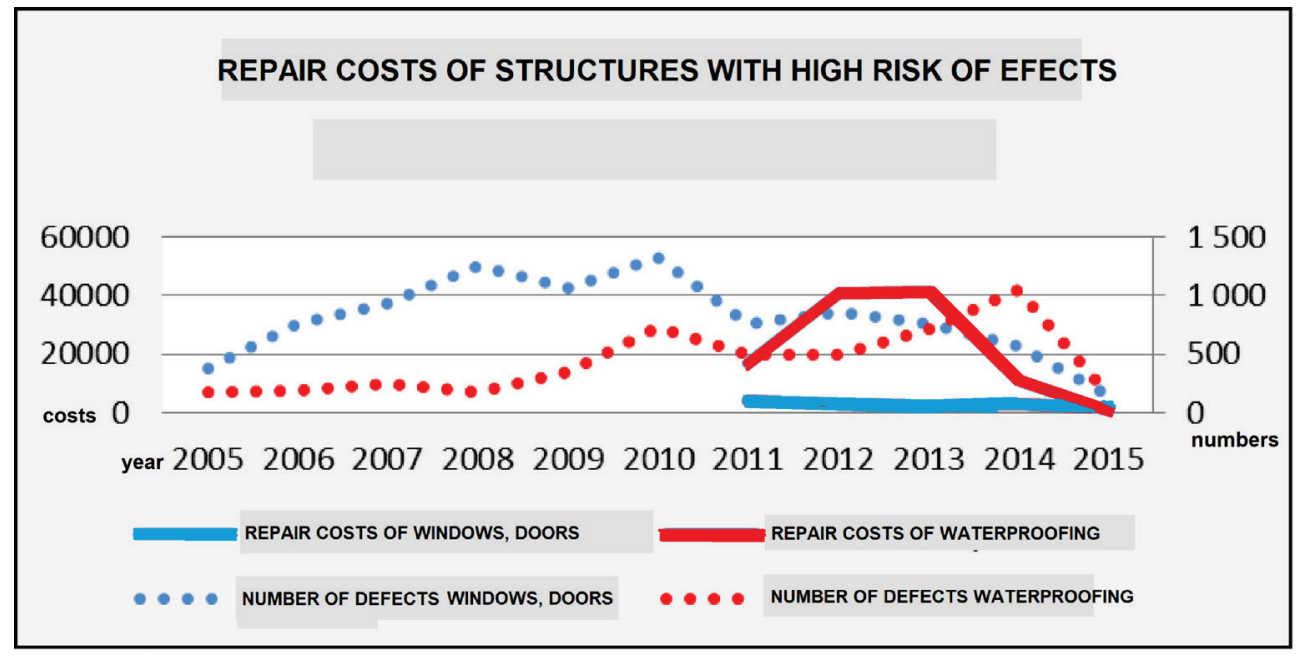

Fig. 3. Decline of development of defects and cost of repairs of high-risk structures y. $2005-2015$ (red dots numbers of waterproofing defects, blue dots number of windows and doors defects /on right/, red costs of waterproofing reapirs, blue costs of windows and doors reapairs /on left/).

\section{References}

1. Oxford Learner's Dictionary. Available on http://www.oxfordlearnersdictionaries.com/definition/english/risk 1?q=risk

2. M. Tichý, Ovládání rizika: analýza a management (C.H. Beck, Prague, 2006)

3. A.D. Hall, A Methodology for Systems Engineering (Van Nostrand Reinhold, 1962)

4. H.F. Cervone, OCLC Systems \& Services: International digital library perspectives 31 (3), 108-111 (2015)

5. D. Baloi, A.D.F. Price, International Journal of Project Management 21, 261-269 (2003) 
